\title{
Diagnostic Accuracy of MR Mammography in Diagnosing Malignant Breast Lesions Taking Histopathology as Gold Standard
}

\author{
Saba Fatima, Shahid Waheed and Muhammad Imran Khan
}

\begin{abstract}
Objective: To find out the diagnostic accuracy of MR Mammography in diagnosing malignant breast lesions taking histopathology as gold standard.

Study Design: Cross-sectional survey.

Place and Duration of Study: Department of Radiology in collaboration with Department of Pathology, Sir Ganga Ram Hospital, Lahore, from April 2015 to April 2016.

Methodology: 150 female patients with either suspicious mammographic findings or palpable lesions suspicious for malignancy referred from surgical OPD of Sir Ganga Ram Hospital were assessed. Dynamic contrast enhanced MRI was done on 1.5 Tesla MRI machine and the images were evaluated on morphological and kinetic basis. MR findings were then compared with preoperative FNAC and biopsy findings.

Results: The mean age of the patients was $52.5 \pm 13.4$ years with maximum patients of age group $46-55$ years. Out of $150,118(78.6 \%)$ patients were found to have malignant lesions on MRM while $116(77 \%)$ patients were proved to have malignant lesions on histopathology. There was $93.9 \%$ sensitivity, $73.5 \%$ specificity, $89.3 \%$ diagnostic accuracy, $92.3 \%$ PPV, and $78.1 \%$ NPV of MR mammography in diagnosing malignant breast lesions taking histopathology as gold standard.

Conclusion: Breast MRI has high sensitivity, specificity, and diagnostic accuracy in diagnosis of malignant breast lesions and must be used as primary imaging tool for proper characterisation of the breast lumps.
\end{abstract}

Key Words: Breast cancer, MR mammography, Dynamic contrast enhancement.

\section{INTRODUCTION}

Breast cancer is the most common female malignancy in both the developing and developed world, and the primary cause of death among women globally. In the year 2011, incidence rates of breast cancer were proved to be high in developed regions of the world (>80 per 100,000$)$ and low in most of the developing regions $\left(<40\right.$ per 100,000). ${ }^{1}$ In Pakistan, it was found to be the most common malignancy accounting for $34.6 \%$ of female cancers, ${ }^{2}$ with at least 90,000 women suffering every year. ${ }^{3}$

Radiological assessment with mammography, ultrasound, and MR mammography is a part of triple assessment of breast cancer. Mammography is the primary imaging technique for detection of early breast cancer; however, it has variable sensitivity and specificity ranging from 68$88 \%$ and $82-98 \%$, respectively. 4 Magnetic resonance mammography is a more sensitive tool for detection of breast cancer. However, there is a controversy about the accuracy of MR mammography with sensitivity being as

Department of Radiology and Medical Imaging, Fatima Jinnah Medical University and Sir Ganga Ram Hospital, Lahore, Pakistan

Correspondence: Prof. Shahid Waheed, Department of Radiology and Medical Imaging, Fatima Jinnah Medical University and Sir Ganga Ram Hospital, Lahore, Pakistan E-mail:drswaheed@hotmail.com

Received: November 01, 2017; Accepted: October 13, 2018 high as $88-100 \%$ while its specificity is low being equivalent to that of mammography, i.e. $68-96 \% .5,6 \mathrm{In}$ the year 2012, sensitivity of MR mammography was proved to be $71 \%$ with a specificity of $68 \% .^{7}$ Role of MRI has been established in assessment of post-surgical changes, $, 8,9$ breast implant integrity, ${ }^{10}$ and assessment of neo adjuvant therapy. ${ }^{11}$ Breast MRI has the ability of providing three dimensional spatial information and better visual differentiation of lesions from normal breast tissue based on differences in vascularity and permeability of lesions. ${ }^{12}$ In MRI, lesions are described according to their enhancement patterns, their size, margins, distribution and description of kinetic curve with initial peak and delayed phases of contrast enhancement. ${ }^{13}$

Keeping in view the confliction in previous studies and lack of enough data available for Pakistani population, objective of this study was to determine the diagnostic accuracy of magnetic resonance mammography in diagnosing malignant breast lesions taking histopathology as gold standard.

\section{METHODOLOGY}

This cross-sectional study was conducted in Department of Radiology and Medical Imaging, Sir Ganga Ram Hospital, between April 2015 to April 2016. A total number of 150 female patients were included in the study, who either had suspicious mammographic abnormality or were referred from surgical OPD with breast lumps suspicious for malignancy. The patients 
who were known to have malignant lesions, underwent surgery or chemotherapy, were excluded from the study. Histopathological analysis was done after FNAC or preoperative biopsy of the lumps.

Dynamic contrast enhanced MR (DCE-MRI) imaging was done on 1.5 Tesla MRI unit (vantage Atlas Z, Toshiba, Medequips, Japan). All patients were scanned in prone position with simultaneous examination of both breasts using double breast coil with application of gentle compression in order to minimise motion effects. Prior to positioning, an intravenous line was secured with 20-22 guage cannula with three-way stopcock for optimal contrast injection.

Fat suppression, subtraction and three-dimensional MIP (maximum intensity projection) imaging techniques were employed to generate thin slices with multiplanar reconstruction. Sequences which were acquired included axial T2W STIR (TR/TE 6000/48, flip angle $90^{\circ}$ ), sagittal T2W Fat-Sat (TR/TE $7300 / 80$, flip angle $90^{\circ}$ ), axial 3D T1W with fat suppression (TR/TE 313/4, flip angle $70^{\circ}$ ) and axial $3 \mathrm{D}$ T1W dynamic contrast enhanced GRE with fat suppression (TR/TE 5/2.5, flip angle $15^{\circ}$ ). For contrast enhanced sequences, MRI contrast agent magnevist (dimeglumine gadopentetate, Bayer) was injected intravenously in a dose of $0.2 \mathrm{ml}$ per $\mathrm{kg}$ followed by saline flush of $20 \mathrm{ml}$ and the scan was commenced 20 seconds after contrast injection. Four post-contrast scans were obtained dynamically with total duration of dynamic sequence of 7 minutes 35 seconds. After completion of study, subtraction images were obtained on a pixel by pixel base.

These scans were reviewed on PACS workstation on the basis of morphological and kinetic features. Morphological assessment included margins of lesions (regular or spiculated) and type of enhancement (ductal, peripheral, homogenous). For kinetic analysis, ROI (region of interest) was drawn on enhancing lesion and time-signal intensity curve was plotted with the help of software. These curves demonstrate initial enhancement (within first two minutes), and later enhancement behaviour. Three types of curves were generated; type I with continuous increased enhancement, type II with plateau phase of enhancement and type III with progressive decline in enhancement.

MRM diagnosis of malignancy was based on spiculated borders, peripheral rim or ductal pattern of enhancement with demonstration of type II or III kinetic curves. 150 patients had their preoperative FNAC and core biopsy done after wards which revealed 116 malignant lesions and 34 benign lesions.

Using SPSS version 20, frequencies and percentages of malignant and benign lesions were calculated according to their morphological and kinetic features. Using crosstabulations and a $2 \times 2$ table, these features were compared with histopathological findings and then the diagnostic accuracy in terms of sensitivity, specificity, PPV, and NPV were calculated for both features.

\section{RESULTS}

The age of the patients included in this study ranged from 35 to 85 years with maximum number of patients falling between $46-55$ years $(n=51,34 \%)$ and average age being $52.5 \pm 13.4$ years. Out of total 150 patients, $100 \%, \quad(n=150), 78.6 \% \quad(n=118)$ were found having malignant breast lesions on MRM with $21.3 \% \quad(n=32)$ having benign lesions. There were $77 \% \quad(n=116)$ malignant lesions while $22 \%(n=34)$ benign lesions on histopathology. On DCE-MRI morphologically, there were 116 lesions with spiculated borders, out of which 69 had ductal enhancement $(59.4 \%)$ while 47 had peripheral enhancement (40.5\%) (Table I). Eighty-six patients displayed type III wash out curve, 35 displayed type II plateau curve and type I curve was seen in 19 (29\%).

Sensitivity, specificity, positive predictive value and negative predictive value of MRM in diagnosing malignant breast lesions came out to be $93.9 \%, 73.5 \%$, $92 \%, 78 \%$ with diagnostic accuracy of $89.3 \%$ (Table II).

Table I: Frequency and percentage of various morphological features assessed on MRI.

\begin{tabular}{lcc}
\hline & Frequency & Percentage \\
\hline Spiculated borders, ductal enhancement & 69 & 46.0 \\
Spiculated borders, peripheral enhancement & 47 & 31.3 \\
Regular borders, no enhancement & 20 & 13.3 \\
Regular borders, homogenous enhancement & 14 & 9.3 \\
\hline Total & 150 & 100.0 \\
\hline
\end{tabular}

Table II: Breast lesions according to MRI and histopathology.

\begin{tabular}{l|c|c|c}
\hline \multirow{2}{*}{$\begin{array}{l}\text { MR mammographic } \\
\text { findings }\end{array}$} & \multicolumn{2}{|c|}{ Histopathogical findings } & \multirow{2}{*}{ Total } \\
\cline { 2 - 3 } Malignant (+ve) & $109(\mathrm{a})$ & $9(\mathrm{~b})$ & $118(78.6 \%)$ \\
\hline Benign (-ve) & $7(\mathrm{c})$ & $25(\mathrm{~d})$ & $32(21.3 \%)$ \\
\hline Total & $116(77.3 \%)$ & $34(22.6 \%)$ & $150(100 \%)$ \\
\hline Sensitivity & $\mathrm{a} / \mathrm{a}+\mathrm{c}=109 / 116=93.9 \%$ & & \\
\hline Specificity & $\mathrm{d} / \mathrm{b}+\mathrm{d}=25 / 34=73.5 \%$ & & \\
\hline PPV & $\mathrm{a} / \mathrm{a}+\mathrm{b}=109 / 118=92.3 \%$ & & \\
\hline NPV & $\mathrm{d} / \mathrm{c}+\mathrm{d}=25 / 32=78.1 \%$ & & \\
\hline Accuracy & $\mathrm{a}+\mathrm{d} / \mathrm{a}+\mathrm{b}+\mathrm{c}+\mathrm{d}=109+25 / 150$ & & \\
& $=89.3 \%$ & & \\
\hline
\end{tabular}

\section{DISCUSSION}

Breast cancer is the most common female cancer worldwide and in Pakistan. Pakistan ranking second in Asian countries. ${ }^{3}$ Multimodality imaging is used for diagnosis and staging of breast cancer. Conventional mammography is the initial screening tool for breast malignancy but because of limitations of variable diagnostic accuracy and decreased performance in dense breasts, ${ }^{14} \mathrm{MRI}$ breast is becoming popular as a new imaging tool. It provides structural and functional details of the lesions based on their vascular characteristics. ${ }^{15}$ Malignant lesions have spiculated borders with ductal/peripheral enhancement, ${ }^{16}$ and type III kinetic curve. ${ }^{17}$

In this study, 118 patients were found to have malignant lesions on MRI breast, while 116 patients had malignancy 
confirmed on histopathology. Out of those $118,77 \%$ lesions had spiculated borders with $46 \%$ ductal and $31 \%$ peripheral enhancement while type III kinetic curve was present in $57 \%$ and type II curve in $23 \%$. Diagnostic accuracy of MR mammography had been variable in different studies with high sensitivity of order of $95 \% 18$ and $71 \%,{ }^{7}$ while specificity is stated as $68 \%{ }^{7}$ and $75 \% .{ }^{19}$ A study conducted at the Aga Khan University proved sensitivity, specificity, PPV, and NPV to be $94 \%, 85 \%$, $90 \%$, and $82 \%$, respectively with overall accuracy of MR mammogram $90 \% .20$ In this study, sensitivity, specificity, PPV, and NPV of MR mammography came out to be $93.9 \%, 73.5 \%, 92.3 \%$ and $78.1 \%$ with $p$-value $<0.001$. The overall diagnostic accuracy to be proved as 89.3 in diagnosing malignant breast lesions. In light of these statistical analysis, MR mammography is recommended to be used in preoperative disease characterisation and staging with high level of diagnostic accuracy.

Limitation of this study included high cost of the investigation and contrast material, increased scan time and prone position, making it uncomfortable for the patients and the referral of only those patients who had suspicious lumps; so there were more patients with malignant lesions. Hence, the patient was hospitalbased and biased. These results and pattern point out towards a judicious and selective use of the modality in breast cancer patients.

\section{CONCLUSION}

Breast MRI is highly accurate for diagnosis of breast carcinoma in terms of sensitivity, specificity and overall diagnostic accuracy in contrast to previous studies which showed decreased specificity. Hence, it should be used as a first line imaging modality for proper characterisation of any breast lesion.

\section{REFERENCES}

1. Curado MP. Breast cancer in the world: Incidence and mortality. Salud Publica Mex 2011; 53:372-84.

2. Shaukat U, Ismail M, Mehmood N. Epidemiology, major risk factors and genetic predisposition for breast cancer in the Pakistani population. Asian Pac J Cancer Prev 2013; 14:5625-9.

3. Asif HM, Sultana S, Akhtar N, Rehman JU, Rehman RU. Prevalence, risk factors and disease knowledge of breast cancer in Pakistan. Asian Pac J Cancer Prev 2014; 15:4411-6.

4. Svahn TM, Chakraborty DP, Ikeda D, Zackrisson S, Do Y, Mattsson $\mathrm{S}$, et al. Breast tomosynthesis and digital mammography: A comparison of diagnostic accuracy. BJR 2012; 85:1074-82.

5. Lee SH, Cho N, Kim SJ, Cha JH, Cho KS, Ko ES, et al. Correlation between high resolution dynamic MR features and prognostic factors in breast cancer. Korean J Radiol 2008; 9:10-8.
6. Agner SC, Soman S, Libfeld E, McDonald M, Thomas K, Englander $S$, et al. Textural kinetics: A novel dynamic contrastenhanced (DCE)-MRI feature for breast lesion classification. J Digit Imaging 2011; 24:446-63.

7. Hillman BJ, Harms SE, Stevens G, Stough RG, Hollingsworth AB, Kozlowski KF, et al. Diagnostic performance of a dedicated 1.5-T breast MR imaging system. RSNA 2012; 265:52-8.

8. Margolis NE, Morley C, Lotfi P, Shaylor SD, Palestrant S, Moy $L$, et al. Update on imaging of the postsurgical breast. Radiographics 2014; 34:642-60.

9. Juanpere S, Perez E, Huc O, Motos N, Pont J, Pedraza S. Imaging of breast implants-a pictorial review. Insights Imaging 2011; 2:653-70.

10. Colombo G, Ruvolo V, Stifanese R, Perillo M, Garlaschi A. Prosthetic breast implant rupture: imaging-pictorial essay. Aesthet Plastic Surg 2011; 35:891-900.

11. Orel SG, Schnall MD. MR imaging of the breast for the detection, diagnosis, and staging of breast cancer. Radiology 2001; 220:13-30.

12. Bhooshan N, Giger ML, Jansen SA, Li H, Lan L. Cancerous breast lesions on dynamic contrast-enhanced MR images: Computerized characterization for image-based prognostic markers. Radiology 2010; 254:680-90.

13. Partridge SC, Mullins CD, Kurland BF, Allain MD. Apparent diffusion coefficient values for discriminating benign and malignant breast MRI lesions: Effects of lesion type and size. AJR 2010; 194:1664-73.

14. Robbins J, Jeffries D, Roubidoux M, Helvie M. Accuracy of diagnostic mammography and breast ultrasound during pregnancy and lactation. Am J Roentgenol 2011; 196:716-22.

15. Yabuuchi H, Matsuo Y, Okafuji T, Kamitani T, Soeda H, Setoguchi $\mathrm{T}$, et al. Enhanced mass on contrast-enhanced breast MR imaging: Lesion characterization using combination of dynamic contrast-enhanced and diffusion-weighted MR images. J Magn Reson Imaging 2008; 28:1157-65.

16. Newell D, Nie K, Chen JH, Hsu CC, Hon JY, Nalcioglu O, et al. Selection of diagnostic features on breast MRI to differentiate between malignant and benign lesions using computer-aided diagnosis: differences in lesions presenting as mass and nonmass-like enhancement. Eur Radiol 2010; 20:771-81.

17. El Khouli RH, Macura KJ, Jacobs MA, Khalil TH, Kamel IR, Dwyer A, et al. Dynamic contrast-enhanced MRI of the breast: quantitative method for kinetic curve type assessment. Am J Roentgenol 2009; 193:W295-300.

18. Kneeshaw PJ, Turnbull LW, Drew PJ. Current applications and future direction of MR mammography. Br J Cancer 2003; 88: 4-10.

19. Bogner W, Gruber S, Pinker K, Grabner G, Stadlbauer A, Weber $\mathrm{M}$, et al. Diffusion-weighted MR for differentiation of breast lesions at $3.0 \mathrm{~T}$ : How does selection of diffusion protocols affect diagnosis? Radiology 2009; 253:341-51

20. Shafqat G, Agha A, Masror I, Rehan M, Afzal S. Dynamic contrast enhanced MRI breast for lesion detection and characterization with histopathological correlation: Preliminary experience at tertiary care hospital. J Pak Med Assoc 2011; 61:252. 